\section{Estudo \\ Ecidebate}

em Testão

Plamejamento
Revista Estudo \& Debate, Lajeado, v. 25, n. 3, 2018. ISSN 1983-036X

DOI: http://dx.doi.org/10.22410/issn.1983-036X.v25i3a2018.1517

\title{
MITIGANDO O PRECONCEITO DO ESTUDO DE CASO QUALITATIVO NA GESTÁO PELA ADOÇÁO DOS CRITÉRIOS DE QUALIDADE
}

\author{
Cláudia Aparecida Avelar Ferreira ${ }^{1}$
}

\begin{abstract}
Resumo: No âmbito da pesquisa sobre gestáo, o estudo de caso como ferramenta de estratégia de pesquisa é estigmatizado por alguns pesquisadores internacionais, porém é muito utilizado pelos brasileiros, o que leva ao pensamento de que esse método necessita de bom delineamento e parâmetros de qualidade, desde o levantamento do problema até o encerramento da análise. O objetivo basilar deste ensaio teórico foi minimizar o motivo que leva ao estigma do estudo de caso no meio acadêmico internacional e apresentar alternativas para minimizar o preconceito com o uso dessa ferramenta em estudos sobre gestáo. Isso deve ser desconstruído antes de se fazer a opçáo por essa estratégia, pois os argumentos se referem à falta de generalizaçáo dos resultados e a discussão dos dados. Essa análise leva a reflexáo de que, ao escolher a estratégia de estudo de caso, o pesquisador deve aprofundar seus conhecimentos sobre esse tipo de estudo, conhecer seus desafios e garantir a confiabilidade e validade do constructo, interna e externamente, a partir das ações descritas no estudo. O preconceito ao uso do estudo de caso tem diminuído nos últimos anos, porém percebe-se discriminação, ainda, em periódicos internacionais devido à visão dominante positivista.
\end{abstract}

Palavras-chave: Estudo de caso. Pesquisa qualitativa. Critérios de qualidade. Projeto de pesquisa.

\section{MITIGATING THE PREJUDICE OF THE QUALITATIVE CASE STUDY IN THE MANAGEMENT BY ADOPTING THE QUALITY CRITERIA}

\begin{abstract}
In the context of management research, the case study as a research strategy tool is stigmatized by some international researchers, but is widely used by Brazilians, which leads to the idea that this method needs good delineation and quality parameters, since the problem until the end of the analysis. The basic objective of this theoretical essay was to minimize the reason that leads to the stigma of the case study in the international academic environment and to present alternatives to minimize prejudice with the use of this tool in management studies. This must be deconstructed before making the choice for this strategy, since the arguments refer to the lack of generalization of the results and the discussion of the data. This analysis leads to the reflection that, in choosing the case study strategy, the researcher must deepen his knowledge about this type of study, know its challenges and guarantee the reliability and validity of the construct, internally and externally, from the actions described in study. Prejudice to the use of the case study has diminished in the last years, but it is perceived discrimination, still, in international periodicals due to the dominant positivist vision.
\end{abstract}

Keywords: Case study. Qualitative research. Quality criteria. Research project.

1 Doutoranda em Administração (PUCMINAS). Mestre em Administração (Centro Universitário UNA). 


\section{INTRODUÇÁO}

Considerando-se a popularidade da estratégia metodológica do estudo de caso, um dos desafios para os pesquisadores no âmbito da gestão é continuar utilizando esse método devido à baixa credibilidade percebida por alguns pesquisadores em não considerá-lo científico dentro dos critérios padronizados da ciência, levando a uma ampla discussão no sentido do rigor metodológico e do plano de pesquisa (LIMA; ANTUNES; MENDONÇA NETO; PELEIAS, 2012; MARQUES; CAMACHO; ALCANTARA, 2015;LARRINAGA, 2017).

No âmbito internacional os pesquisadores positivistas refutavam esse método, porém observa-se uma mudança de postura nos últimos tempos. Os autores Harrison, Birks, Franklin e Mills (2017) argumentam que houve um grande progresso nos últimos quarenta anos no desenvolvimento metodológico do estudo de caso, gerando uma abordagem pragmática e flexível e sendo aplicado em diversas disciplinas, ocasionando distintas definições, aplicação e validade. Freitas e Jabbour (2011), Lima et al. (2012) e Marques et al. (2015) enfatizam que na administração e contabilidade é muito utilizado o estudo de caso, sendo que os pesquisadores brasileiros na esfera da gestáo passaram a utilizar de forma intensiva esse método a partir dos anos 80 , o que não corresponde com a visão acadêmica anglo-saxônica (MARIOTTO; ZANNI; MORAES, 2014).

O estudo de caso é muito utilizado no desenvolvimento das áreas de estratégia e gestão devido à singularidade e a conjectura do caso com o envolvimento de diversas fontes de dados (EISENHARDT, 1989; VOSS; TSIKRIKTSIS; FROHLICH, 2002; CEPEDA; MARTIN, 2005; YIN, 2005/2009; MARTINS, 2008; RIDDER; HOON; MCCANDLESS, 2009; FREITAS; JABBOUR, 2011; LIMA et al., 2012; MARQUES et al., 2015; ZAPPELLINI; FEUERSCHÜTTE, 2015). No entanto, Cepeda e Martin (2005) ressaltam que o uso de estudo de caso em pesquisa qualitativa com possibilidade de construção de teoria é uma lacuna nos trabalhos já publicados e tornou-se uma possibilidade para fazer um plano de estudo de caso rigoroso. As áreas que mais utilizam essa ferramenta são as ciências sociais aplicadas, a saúde, a contabilidade, a gestão e o planejamento urbano (LIMA et al., 2012; MARIOTTO et al, 2014; MARQUES et al., 2015, HARRISON et al., 2017). Foi adotada neste artigo a definição para estudo de caso de Yin (2003, p. 13), que define o método como "uma investigação empírica que investiga um fenômeno contemporâneo dentro de seu contexto de vida real, especialmente quando as fronteiras entre fenômeno e o contexto não são claramente evidentes”.

Para minimizar o preconceito, o pesquisador deve estar apto para otimizar o estudo de caso, utilizando os critérios de qualidade aplicados a esse método, ou refutá-los. Compreendese como validade do constructo, a qualidade do framework teórico e as operaçóes entre constructos importantes em uma pesquisa ou o uso de métricas adequadas para aplicação aos constructos em estudo, no caso de estudo quantitativo (EISENHARDT, 1989; YIN, 2005, 2009; DE MASSIS; KOTLAR, 2014; MARQUES et al., 2015; LARRINAGA, 2017). No estudo de caso qualitativo, a teoria está alinhada com o fenômeno, logo, em bases sólidas empíricas (YIN, 2005, 2013; FREITAS; JABBOUR, 2011; WELCH et al., 2011, LIMA et al., 2012; MARQUES et al., 2015; LARRINAGA, 2017). 
Marques et al. (2015) analisaram 180 artigos delineados como estudo de caso, em periódicos do âmbito da contabilidade gerencial, no período compreendido de 2008 a 2012, classificação Qualis Capes A2, B1 e B2, utilizando como meio 15 critérios de qualidade para identificar o rigor metodológico. As falhas encontradas nos estudos foram em decorrência de: baixa evidência ao justificar a importância do fenômeno no contexto do estudo; inexistência de explicação da escolha do método; objetivos pouco esclarecedores que não proporcionam o aprofundamento da análise; utilização de poucas fontes de evidências; baixo uso da triangulação de dados e informaçóes; falta de prioridade no relato da coleta de dados; vários estudos ambíguos que não esclarecem se o estudo de caso é método ou coleta de dados; poucos artigos que não informaram como ocorreu a análise de dados; baixa ênfase nas contribuiçôes do estudo de caso; e raros citaram as limitaçóes do estudo sugerindo continuidade.

Outro estudo como dos autores Castro e Rezende (2018) que analisaram 206 artigos publicados no Brasil no período de 2004 a 2013, buscou verificar os critérios de qualidade nos estudos de caso. Foram identificadas açóes de validade do constructo e validade interna relacionada com a classificação dos periódicos e não encontraram açóes de validade externa e confiabilidade, demonstrando a necessidade de maior rigor nos estudos qualitativos.

Portanto, o problema em questão é que a maioria dos estudos de casos qualitativos desenvolvidos no Brasil não aplica ou se aplica não insere no artigo os critérios de qualidade necessários para se obter reconhecimento internacional, como ocorre com os estudos de casos quantitativos, que tendem a demonstrar sua robustez. Náo é um tema novo na academia, mas a proposta dá maior amplitude aos estudos de casos qualitativos do Brasil, que ainda não preenche a lacuna em relação ao rigor exigido como nos estudos quantitativos, conforme identificado nos estudos de Marques et al. (2015) e Castro e Rezende (2018).

Os critérios de qualidade ressaltados neste estudo foram: a validade interna tem como fundamentação a relação causal entre os constructos ou variáveis por questóes naturais (EISENHARDT, 1989; LEE, 1989; YIN, 2005; GIBBERT; RUIGROK; WICKI, 2008; WELCH et al.,2011; LIMA et al., 2012; MARQUES et al., 2015; EISENHARDT; GRAEBNER; SONENSHEIN, 2016) sendo mais utilizada em estudo explanatório (YIN, 2005, 2013; WELCH et al., 2011). A validade externa refere-se à generalização das conclusōes da pesquisa. A generalização analítica ou teórica ocorre na extrapolação dos achados empíricos para outros estudos mais abrangentes e contextos diferentes (GIBBERT et al., 2008; RUZZENE, 2012; LIMA et al., 2012; YIN, 2013; MARQUES et al., 2015; EISENHARDT et al., 2016). Também, importante é a confiabilidade dos documentos da pesquisa e da transparência nos procedimentos (LIMA et al., 2012; DE MASSIS; KOTLAR, 2014; MARQUES et al., 2015).

Diante do exposto, indaga-se porque alguns autores tendem a estigmatizar o estudo de caso. O objetivo basilar deste ensaio teórico foi desmitificar o estigma do estudo de caso qualitativo brasileiro no meio acadêmico internacional e apresentar alternativas para minimizar o preconceito com essa ferramenta na gestão. Desta forma, os autores estarão preparados para que todo o seu esforço náo seja perdido no momento que decidam publicar seu estudo internacionalmente ou em um periódico nacional com classificação significativa da CAPES. Para tanto, foi utilizada como estratégica metodológica a revisão de literatura. 


\section{ESTUDO DE CASO}

No âmbito da gestão, a utilização do estudo de caso é uma ferramenta que facilita gerar e testar teoria e tem despertado interesse nos acadêmicos, bem como nos editores de revistas de gestão no Brasil. É aplicado em diversas ciências como na antropologia, psicologia, educação, medicina, direito, administração, ciência política, dentre outras (WELCH et al., 2011; LIMA et al, 2012; MARIOTTO et al., 2014; TSANG, 2014; MARQUES et al., 2015; LARRINAGA, 2017). Apresenta significaçóes diferentes devido à variedade de campos de estudo podendo ser um método de investigação, uma abordagem metodológica, um projeto de pesquisa ou uma abordagem monográfica, dentre outros (MARIOTTO et al., 2014). Segundo Mariotto et al. (2014, p.359), estudo de caso é uma "descrição detalhada de uma situação de gestão”.

Os usos e significados do método apresentam diversas implicaçóes de grande amplitude para a conduta das ciências sociais. $\mathrm{O}$ estudo de caso pode ser usado em pesquisas qualitativas e quantitativas (RAGIN; BECKER, 1992; YIN, 1981, 2009; FREITAS; JABBOUR, 2011; WELCH; PIEKKARI; PLAKOYIANNAKI; PAAVILAINEN-MÄNTYMÄKI, 2011; LIMA et al., 2012; MARIOTTO et al, 2014; MARQUES et al., 2015, HARRISON et al., 2017). O estudo de caso qualitativo é estigmatizado e sofre preconceito, o que tem dificultado o desenvolvimento conceitual (RAGIN; BECKER, 1992; CEPEDA; MARTIN, 2005; WELCH et al., 2011, LIMA et al, 2012, MARQUES et al., 2015; LARRINAGA, 2017) devido à falta de rigor metodológico em pesquisas qualitativas. Os casos podem ser empíricos, reais e limitados, mas específicos e também casos como objetos porque são gerais e convencionais (RAGIN; BECKER, 1992).

Diante da pergunta: O que é um caso? Muitos cientistas sociais antes de 1980 não tinham um consenso quanto à definição desta questão. Um caso pode ser teórico (incorporado) ou empírico (holístico), ou ambos; pode ser um objeto relativamente limitado ou um processo, pode ser genérico e universal (múltiplo) ou específico (único) de alguma forma. Ainda pode ser típico ou exemplar ou teoricamente decisivo em outro contexto. No estudo de caso, o princípio da repetição muitas vezes está implicado em afirmaçóes sobre a relação entre o caso escolhido e outros casos (RAGIN; BECKER, 1992; YIN, 2005, 2014; FREITAS; JABBOUR, 2011; LIMA et al., 2012; MARQUES et al., 2015; LARRINAGA, 2017). Yin (1981) delimita o estudo de caso partindo do holístico para o incorporado e do singular para o múltiplo, enquanto Ragin (1992) parte das unidades empíricas para construçóes teóricas e do específico para o geral, mas ambos correspondem à mesma estrutura, porém com denominaçóes diferentes.

Já para Yin (1981), Lima et al. (2012) e Marques et al. (2015), o estudo de caso é uma estratégia de pesquisa que visa alinhar a conjuntura do contexto da vida real sobre o fenômeno, ao mesmo tempo. Isto pode ser confrontado através de um experimento, uma história ou uma simulação, que podem ser consideradas estratégias de pesquisa alternativas. Apresenta uma característica específica que é pesquisar o fenômeno na contemporaneidade, quando as fronteiras entre o fenômeno e o contexto não são evidentes. A associação da estratégia proposta com método de coleta de dados e as provas sáo o cerne da questão para realizar o estudo de caso. 
Um bom estudo de caso deve ser delineado no projeto de pesquisa. As perguntas da pesquisa devem buscar explicaçóes e náo questóes de incidência; e os tipos de projetos de estudo de caso devem envolver o problema essencial, porque o contexto é parte do estudo. A estratégia quanto aos estudos de caso, pode ser exploratória, descritiva e explicativa (YIN, 1981; LIMA et al., 2012; MARQUES et al., 2015).

\section{PROJETO DE PESQUISA}

Para Yin (2005), Lima et al. (2012), Marques et al. (2015) e Larrinaga (2017), o projeto de pesquisa que utiliza a estratégia de estudo de caso, seja único ou múltiplo, deve refletir situaçóes diferenciadas, além de ter unidades de análises unitárias ou múltiplas. Compreende-se por projeto de pesquisa um plano de atividades que devem ter quatro questôes: "quais problemas estudar, quais dados são relevantes, quais dados coletar e como analisar os resultados" (YIN, 2005, p.41).

O processo inicia-se com a definição do problema, segundo YIN (2005) e MOHD NOOR (2008), a validaçáo do constructo, replicação (essencial para análise de casos múltiplos), validade, confiabilidade (YIN, 1981, 1984, 2014; LIMA et al., 2012; MARQUES et al., 2015) e triangulação dos achados (JICK, 1979; EISENHARDT, 1989; STAKE, 1995; VOSS et al., 2002; YIN, 2005; MARTINS, 2008; GIBBERT; RUIGROK, 2010; FREITAS; JABBOUR, 2011; ZAPPELLINI; FEUERSCHÜTTE, 2015; LIMA et al., 2012; MARQUES et al., 2015). Eisenhardt (1989) enfatiza que o método se baseia na comparação de dados de modo contínuo e uso da teoria, desde o início até a coleta de dados. As categorias analíticas surgem a partir da prova teórica e de uma abordagem incremental, para a seleção de casos e de coleta de dados. E para agrupar todas essas fases é necessário um projeto de pesquisa, formado logicamente pela uniáo dos dados a serem coletados, sendo que as conclusốes devem ser baseadas nas perguntas iniciais do estudo (YIN, 2005).

Os itens que compóem um projeto de pesquisa são as questões do estudo; suas proposiçóes (não é obrigatório); as unidades de análise; a lógica que une dados às proposiçóes e os critérios para interpretar as constataçóes. O problema do estudo deve levantar qual estratégia de pesquisa deve ser aplicada (no que se refere a estudo de caso) e iniciar a pergunta usando "como" ou "por que" (YIN, 2005; RIDDER et al., 2009; WELCH et al., 2011; LIMA et al., 2012 ; MARQUES et al., 2015). As proposiçóes devem conduzir ao escopo do estudo. A unidade de análise pode ser única (estudo de caso único) ou múltipla (estudo de caso múltiplo). A unidade de análise refere-se ao dilema de definir o que é um caso, que pode ser indivíduo, programa, escola, comunidades, organizaçóes, dentre outros. No caso do indivíduo, a unidade de análise é primária. A unidade de análise ou estudo de caso deve ser convergente com os estudos anteriores executados por outros pesquisadores, ou divergente, porém, viável no processo operacional (YIN, 2005; RIDDER et al., 2009).

Freitas e Jabbour (2011) corrobora Gibbert e Ruigrok (2010) e Voss et al. (2002) na opiniáo de que os casos únicos são mais difíceis para fazer generalizaçóes na conclusão. Por isso, tolhem o desenvolvimento de modelos e teorias. No entanto, não há um consenso do que seja um estudo de caso único ou unidade de análise, uma vez que essa pode ser uma pessoa, um grupo de pessoas ou mesmo uma política, uma prática cultural, um processo 
de trabalho ou uma estratégia organizacional, sendo que essa acepção de unidade de análise depende do objetivo do pesquisador em abordar o estudo de caso.

A ligação de dados às proposiçôes e os critérios para interpretação das constataçóes são realizados na análise de dados. A ligaçáo das proposiçóes ocorre de maneira que diversas partes da informação coletada podem ser referenciadas à mesma proposição. Na interpretação das constataçóes do estudo, os dados devem se ajustar a um padrão para serem comparados a pelo menos duas proposiçóes concorrentes (YIN, 2005, MARQUES et al., 2015).

$\mathrm{O}$ estudo de caso único deve apresentar alguns requisitos que o distingue do estudo de caso múltiplo como ser decisivo (testa uma teoria bem formulada), raro ou extremo, representativo ou típico, revelador e, por fim, longitudinal (estuda o mesmo caso com dois ou mais pontos diferentes), ou também quando do estudo de caso piloto. Por outro lado, quando o mesmo caso envolve mais de uma unidade de análise, gerando subunidades, é chamado de estudo de caso incorporado versus holístico. O projeto holístico apresenta vantagem quando é inviável identificar subunidade lógica e a teoria é subjacente ao estudo de caso. Em estudo de casos múltiplos, a confecção de esquema facilita o trabalho e deve seguir a lógica de replicação e não de amostragem. Nesse tipo, a escolha dos casos deve ser bem elaborada e os casos devem ser similares aos experimentos múltiplos, com replicação literal ou contraditória, já prevista no princípio do estudo (YIN, 2005).

Para Yin (2005), Gibbert e Ruigrok (2010), Lima et al. (2012) e Marques et al. (2015), a generalização em estudo de caso único é criticada por pesquisadores porque acreditam que os dados são pobres, não possibilitando fazer generalizações estatísticas, sendo que no estudo de caso as generalizaçóes sáo analíticas. Nos casos de projetos holísticos com estudo de caso único por inteiro pode acontecer abstração, falta de dados ou métricas claras. A situação de projeto incorporado ocorre quando o estudo de caso se concentra somente na esfera de subunidades e não consegue retornar à unidade de análise maior.

Para aumentar o rigor nos estudos de casos segundo Eisenhardt (1989), Yin (2009) Lima et al. (2012), Marques et al. (2015) e Larrinaga (2017) faz-se necessário implementar estratégias que assegurem a precisão dos estudos como: validade interna e externa; validade do constructo; e confiabilidade. Eisenhardt (1989) buscou contribuir na construção de uma teoria para o estudo de caso apresentando um roteiro sintético para método qualitativo (Quadro 1) e com o posicionamento teórico de estudos de caso nos contextos das ciências sociais, por explorar forças e fraquezas nesses estudos. 
Quadro 1- Roteiro sintético para método qualitativo

\begin{tabular}{|c|c|c|}
\hline Passos & Atividade & Objetivos \\
\hline Início & $\begin{array}{l}\text { Definição da questão de investigação, } \\
\text { cuja construção é prioridade. }\end{array}$ & $\begin{array}{l}\text { Concentrar ao máximo; } \\
\text { proporcionar melhor fundamento das medidas } \\
\text { de construçáo. }\end{array}$ \\
\hline $\begin{array}{l}\text { Seleção de } \\
\text { caso }\end{array}$ & $\begin{array}{l}\text { Nem teorias, nem hipóteses; } \\
\text { especificidade da população; } \\
\text { amostragem teórica, não aleatória. }\end{array}$ & $\begin{array}{l}\text { Manter a flexibilidade teórica; } \\
\text { excluir variação anormal objetivando a validade } \\
\text { externa; } \\
\text { concentrar esforços em teoria útil para o } \\
\text { caso, ou seja, replicar ou estender a teoria, } \\
\text { preenchendo categorias conceituais. }\end{array}$ \\
\hline $\begin{array}{l}\text { Elaboração de } \\
\text { instrumentos e } \\
\text { protocolos. }\end{array}$ & $\begin{array}{l}\text { Método de coleta de dados múltiplos; } \\
\text { dados combinados qualitativos e } \\
\text { quantitativos; } \\
\text { vários pesquisadores. }\end{array}$ & $\begin{array}{l}\text { Fortalecer a ligação da teoria com os protocolos } \\
\text { - triangulaçáo; } \\
\text { buscar o sinergismo; } \\
\text { promover perspectivas divergentes e reforçar a } \\
\text { ligaçáo da teoria com os achados. }\end{array}$ \\
\hline $\begin{array}{l}\text { Pesquisa de } \\
\text { campo }\end{array}$ & $\begin{array}{l}\text { Sobreposição de coleta de dados e } \\
\text { análises, incluindo notas de campo; } \\
\text { métodos flexíveis e oportunistas de } \\
\text { rejeitar dados. }\end{array}$ & $\begin{array}{l}\text { Analisar rapidamente para fazer os ajustes } \\
\text { necessários para coleta de dados. } \\
\text { Permitir que os pesquisadores extraiam os } \\
\text { temas emergentes e as características únicas do } \\
\text { processo. }\end{array}$ \\
\hline $\begin{array}{l}\text { Análise de } \\
\text { dados }\end{array}$ & $\begin{array}{l}\text { Análise intracaso; } \\
\text { Pesquisa de caso transversal usando } \\
\text { diferentes técnicas. }\end{array}$ & $\begin{array}{l}\text { Familiarizar com os dados e processar para } \\
\text { geração de teoria; } \\
\text { Visão holística dos pesquisadores buscando ver } \\
\text { provas através dos dados. }\end{array}$ \\
\hline $\begin{array}{l}\text { Modelagem de } \\
\text { hipóteses }\end{array}$ & $\begin{array}{l}\text { Tabulação individual dos constructos; } \\
\text { Replicação, sem amostragem, em todos } \\
\text { os casos; } \\
\text { Pesquisar o "porquê" detrás dos } \\
\text { relacionamentos. }\end{array}$ & $\begin{array}{l}\text { Reforça a definição do constructo, validade e } \\
\text { medição; } \\
\text { Confirmar, estender, e reforça a teoria; } \\
\text { Construir a validade interna. }\end{array}$ \\
\hline $\begin{array}{l}\text { Envolvendo a } \\
\text { literatura }\end{array}$ & $\begin{array}{l}\text { Comparar com a literatura conflitante; } \\
\text { Comparar com literatura compatível ao } \\
\text { seu achado. }\end{array}$ & $\begin{array}{l}\text { Construir validade interna, elevar o nível teórico } \\
\text { e favorecer a construção de definiçóes; } \\
\text { Favorecer a generalização, melhorar a definição } \\
\text { do constructo e elevar o nível teórico. }\end{array}$ \\
\hline Fechamento & Saturação teórica quando possível. & $\begin{array}{l}\text { Fim do processo quando a melhoria marginal se } \\
\text { torna pequena. }\end{array}$ \\
\hline
\end{tabular}

Fonte: Eisenhardt (1989, p.533).

\section{CRITÉRIOS DE QUALIDADE}

Bonoma (1985), Eisenhardt (1989), Lee (1989), Leonard-Barton (1990), Yin (2005), Flyvjerger (2006), Gibbert e Ruigrok (2010) e Eisenhardt et al. (2016) concordam que os critérios de qualidade de projetos de estudos de caso devem apresentar pelo menos quatro condições, que são: validade do constructo (BONOMA, 1985; EISENHARDT, 1989; LEE, 1989; LEONARD-BARTON, 1990; YIN, 2005; FLYVJERGER, 2006; GIBBERT et al., 2008; GIBBERT ; RUIGROK, 2010; LIMA et al., 2012 ; MARQUES et al., 2015; EISENHARDT et al., 2016); validade interna (em estudos causais ou explanatórios) 
BONOMA, 1985; LEE, 1989; LEONARD-BARTON, 1990; RAGIN; BECKER, 1992; SAYER, 2000; YIN, 2005; FLYVJERGER, 2006; GIBBERT et al., 2008; GIBBERT; RUIGROK, 2010; WELCH et al., 2011; LIMA et al., 2012 ; MARQUES et al., 2015; EISENHARDT et al., 2016); validade externa (BONOMA, 1985; EISENHARDT, 1989; LEE, 1989; LEONARD-BARTON, 1990; RAGIN ; BECKER, 1992; SAYER, 2000; YIN, 2005; FLYVJERGER, 2006; GIBBERT et al., 2008; GIBBERT; RUIGROK, 2010; RUZZENE, 2012; LIMA et al., 2012; MARQUES et al., 2015); e confiabilidade (BONOMA, 1985; EISENHARDT, 1989; LEE, 1989; LEONARD-BARTON, 1990; YIN, 2005; FLYVJERGER, 2006; GIBBERT et al., 2008; GIBBERT; RUIGROK, 2010; LIMA et al., 2012; MARQUES et al., 2015). A validade do constructo tem por objetivo constituir medidas operacionais corretas para os conceitos que estáo no estudo, por meio de várias fontes de evidências e estabelecer um encadeamento de evidências, além de fazer um relatório do estudo de caso revisado pelos entrevistados-chave, que compóem a fase de coleta de dados (BONOMA, 1985; EISENHARDT, 1989; LEE, 1989; LEONARD-BARTON, 1990; YIN, 2005; FLYVJERGER, 2006; GIBBERT et al., 2008; GIBBERT; RUIGROK, 2010; LIMA et al., 2012; MARQUES et al, 2015; EISENHARDT et al., 2016).

A validade interna relaciona-se com estudos causais e explanatórios devido às inferências resultantes do problema e refere-se à fase de análise de dados (BONOMA, 1985; LEE, 1989; LEONARD-BARTON, 1990; RAGIN; BECKER, 1992; SAYER, 2000; YIN, 2005; FLYVJERGER, 2006; GIBBERT et al., 2008; GIBBERT; RUIGROK, 2010; WELCH et al., 2011; LIMA et al., 2012 ; MARQUES et al., 2015; EISENHARDT et al., 2016), pois promove o controle dos efeitos ambientais e a replicação lógica. A validade externa refere-se à generalização dos achados a outros fenômenos similares, que compóem o projeto de pesquisa. Essa generalização é analítica partindo de um conjunto de resultados para alguma teoria mais abrangente. A confiabilidade busca minimizar os erros e os vieses de um estudo, de forma a tornar as etapas do processo operacional viável e conduzir a pesquisa de forma mais detalhada possível, como se fosse ser submetida a uma auditoria na fase de coleta de dados (BONOMA, 1985; EISENHARDT, 1989; LEE, 1989; LEONARDBARTON, 1990; YIN, 2005; FLYVJERGER, 2006; GIBBERT et al., 2008; RUZZENE, 2012; LIMA et al., 2012 ; MARQUES et al., 2015; EISENHARDT et al., 2016).

Marques et al. (2015), Lima et al. (2012), Gibbert e Ruigrok (2010) corroboram Jick (1979) e Eisenhardt (1989) sobre a importância da triangulação, porém não explicam como fazê-la. A triangulação no interior do método tem por objetivo verificar a validade interna e a confiabilidade, enquanto que entre os métodos, objetiva a validade externa. $\mathrm{O}$ uso de somente um método torna o procedimento qualitativo fraco. Por isso, é importante o uso de vários métodos, o que aumenta a profundidade no estudo do fenômeno. $\mathrm{O}$ uso de multimétodos possibilita o fortalecimento da técnica qualitativa, em decorrência de fortalecer a fraqueza de um meio por outra fonte de dados mais robusta, compensando as forças fracas e fortes. Os métodos podem ser: observação direta e participante, entrevistas semiestruturadas, autorrelato ou dados fornecidos pelos colegas, ou supervisores dos sujeitos do estudo, documentos de arquivo, livro de memórias, jornais e memorandos (JICK, 1979; MARQUES et al., 2015; LARRINAGA, 2017). 
Segundo Freitas e Jabbour (2011), corroborando com o mesmo pensamento de: (Eisenhardt, 1989; Yin, 2005; Voss et al., 2002; Martins, 2008; Lima et al., 2012; e Marques et al., 2015), no processo de triangulação são usadas diversas fontes de evidências e a triangulação dos dados de diferentes fontes aumenta a credibilidade e a confiabilidade dos resultados. Para Zappellini e Feuerschütte (2015) a triangulação propicia a associação de diversos métodos de coleta de dados, populaçóes ou amostras e diferentes abordagens teóricas em tempos distintos para se chegar a uma conclusão do fenômeno pesquisado. Devido aos distintos tipos de triangulação de dados, tem ocorrido mau entendimento entre os pesquisadores sobre a triangulação de dados e metodologia.

No entanto, Llewellyn e Northcott (2007) ressaltam que em algumas etapas ou contextos somente uma única pessoa consegue conhecer e discernir o significado e a importância das questóes organizacionais. Stake (1995) ressalta que a pesquisa qualitativa não exige sempre a triangulação, mas em alguns casos é importante, como nas situações em que os achados são incontestáveis. Achados críticos para uma asseveração e interpretações fundamentais justificam uma triangulação forte, e também nos casos de dados cujas persuasóes do autor exige-se pouco investimento na triangulação (STAKE,1995; ZAPPELLINI ; FEUERSCHÜTTE, 2015).

Portanto, na pesquisa organizacional, o uso da pesquisa qualiquantitativa, tornam os resultados mais consistentes e convergentes devido à triangulação dos dados, por permitir testar ou desenvolver hipóteses para os achados mais divergentes encontrados no contexto, além de propiciar a inovação com métodos não convencionais. Enfim, a triangulação metodológica é equivalente à teórica, pois várias teorias são necessárias para lidar com um problema (JICK, 1979; MARQUES et al., 2015).

No Quadro 2 apresenta-se os critérios de qualidade, os autores que concordam com a utilização da validade e confiabilidade e os críticos da abordagem positivista.

Quadro 2 - Critérios de qualidade para estudo de caso qualitativo

\begin{tabular}{|l|l|}
\hline \multicolumn{1}{|c|}{ Critérios } & \multicolumn{1}{c|}{ Autores que aderem aos critérios. } \\
\hline $\begin{array}{l}\text { Validade do } \\
\text { constructo }\end{array}$ & $\begin{array}{l}\text { Bonoma (1985); Eisenhardt (1989); Lee (1989); Leonard-Barton (1990); Yin (2005); } \\
\text { Flyvjerger (2006); Gibbert et al. (2008); Gibbert e Ruigrok (2010); Lima et al. (2012); } \\
\text { Marques et al. (2015); Eisenhardt et al. (2016); Larrinaga (2017); Castro; Rezende (2018). }\end{array}$ \\
\hline $\begin{array}{l}\text { Validade } \\
\text { interna }\end{array}$ & $\begin{array}{l}\text { Bonoma (1985); Lee (1989); Leonard-Barton (1990); Ragin; Becker (1992); Sayer (2000); } \\
\text { Yin (2005); Flyvjerger (2006); Gibbert et al. (2008); Gibbert; Ruigrok (2010); Welch et } \\
\text { al. (2011); Lima et al., (2012); Marques et al., (2015); Eisenhardt et al. (2016); Larrinaga } \\
\text { (2017); Castro; Rezende (2018). }\end{array}$ \\
\hline $\begin{array}{l}\text { Validade } \\
\text { externa }\end{array}$ & $\begin{array}{l}\text { Bonoma (1985); Lee (1989); Leonard-Barton (1990); Ragin; Becker (1992); Sayer (2000); } \\
\text { Stake(2000);Yin (2005); Flyvjerger (2006); Gibbert et al. (2008); Gibbert; Ruigrok } \\
\text { (2010); Ruzzene (2012); Lima et al., (2012); Mariotto et al. (2014); Marques et al. (2015); } \\
\text { Eisenhardt et al. (2016); Larrinaga (2017); Castro; Rezende (2018). }\end{array}$ \\
\hline Confiabilidade & $\begin{array}{l}\text { Bonoma (1985); Eisenhardt (1989); Lee (1989); Leonard-Barton (1990); Stake (2000); } \\
\text { Yin (2005); Flyvjerger (2006); Gibbert et al. (2008); Gibbert; Ruigrok (2010); Lima et al. } \\
\text { (2012); Mariotto et al. (2014); Marques et al. (2015); Eisenhardt et al. (2016); Larrinaga } \\
\text { (2017); Castro; Rezende (2018). }\end{array}$ \\
\hline
\end{tabular}

Fonte: Elaborado pela autora. 
Para obtenção de um estudo de caso com elevado nível de qualidade, propóe-se, para cada critério de qualidade, os dados resumidos a serem observados pelos pesquisadores conforme o Quadro 3.

Quadro 3 - Critérios de qualidade para estudo de caso qualitativo

\begin{tabular}{|c|c|}
\hline Critérios & Dados observados \\
\hline Validade do constructo & $\begin{array}{l}\text { Utilização de múltiplas fontes de evidências; triangulaçãa de evidências; revisão } \\
\text { de transcriçóes e rascunhos por pares acadêmicos; revisão de manuscritos por } \\
\text { informantes-chave; descrição das circunstâncias de acesso ao caso; menção à } \\
\text { estrutura de tempo da coleta de dados; critérios claros de seleçấo de respondentes } \\
\text { da pesquisa; critérios claros de seleçáo de instrumentos de coleta de dados. }\end{array}$ \\
\hline Validade interna & $\begin{array}{l}\text { Estabelecimento de um framework teórico claro derivado da literatura; utilizaçáo de } \\
\text { múltiplos investigadores; utilização de um conjunto de categorias para a análise de } \\
\text { dados; critérios claros de análise de dados da pesquisa. }\end{array}$ \\
\hline Validade externa & $\begin{array}{l}\text { Critérios claros de seleção de casos; utilizaçáo de múltiplos casos; utilização de } \\
\text { casos incorporados; descriçáa dos contextos dos casos estudados; comparação entre } \\
\text { resultados da pesquisa e teoria; estratégia de replicaçáo de casos. }\end{array}$ \\
\hline Confiabilidade & $\begin{array}{l}\text { Menção aos tópicos abordados no roteiro de entrevista; declaração de objetivos, } \\
\text { proposiçôes e ou questáo de pesquisa; menção ao corpus de pesquisa/análise; testes } \\
\text { dos instrumentos de coleta de dados; elaboração de bancos de dados da pesquisa; } \\
\text { gravação de entrevistas pessoais; transcrição cuidadosa das entrevistas gravadas; } \\
\text { utilização de interavaliadores na codificação de dados; apresentaçấo de extratos de } \\
\text { entrevistas nos relatórios. }\end{array}$ \\
\hline
\end{tabular}

Fonte: Autoria dos dados: Bluhm, Harman, Lee \& Mitchell, 2011; Creswell, 2003; Yin, 1981, 2005, 2013; Eisenhardt, 1989; Jick, 1979; Crowe et al., 2011; Gibbert \& Ruigrok, 2010; Gibbert et al., 2008; Eisenhardt et al., 2016; Meyer, 2001; Pettigrew, 1990; Eisenhardt \& Graebner, 2007; Leonard-Barton, 1990; Ruzzene, 2012; Beverland e Lindgreen 2010; Miles \& Hubermann, 1994; De Massis \& Kotlar; 2014; Silverman, 2006; Moret et al., 2007.

No Quadro 3, os dados observados e os critérios de qualidade são informaçóes que o pesquisador pode adotar quando do planejamento detalhado de seu projeto de pesquisa e que podem facilitar muito o seu trabalho. O cumprimento desses quesitos, ou pelo menos de alguns desses, propiciará um bom estudo de caso qualitativo.

\section{DESAFIOS E OPORTUNIDADES}

A limitação de estudo de caso é muito enfatizada pelos pesquisadores positivistas devido à dificuldade de generalizar as conclusóes, principalmente quando se trata de estudo de caso único, por falta de validade externa, impossibilitando aplicar as conclusóes em outros estudos de caso (MARIOTTO et al., 2014 corrobora YIN, 2009). Larrinaga (2017) acrescenta, ainda, que nas situaçóes em que o pesquisador tem número reduzido de caso, ou vários casos com amostra não significativa, é dificultada a generalização para a construção de teoria por não ter uma base sólida que dê sustentabilidade.

Diante das críticas dos pesquisadores positivistas e não positivistas, os pesquisadores têm buscado cumprir os critérios científicos padronizados com o propósito de aumentar o rigor nos estudos de caso (LIMA et al., 2012 ; MARIOTTO et al., 2014; MARQUES et 
al., 2015; LARRINAGA, 2017), usar o caso para gerar teoria e ou aprimorar uma teoria já existente (MARIOTTO et al., 2014; LARRINAGA, 2017).

Contrapondo, Mariotto et al. (2014) advertem que a generalização na pesquisa qualitativa não é uma meta necessária e que o objetivo fim do estudo de caso não é representar o mundo e sim representar o caso (STAKE, 2000; PLATT, 2007). Segundo Stake (2000), o estudo de caso único induz a uma compreensão mais exata das condiçóes em que aconteceu o fenômeno, tornando-o mais rigoroso.

Além disto, os desafios da estratégia de estudo de casos decorrem do processo de avaliação do manuscrito nos periódicos, que pode divergir das concepçóes dos revisores, necessitando, portanto, de um argumento forte que justifique a escolha da pesquisa qualitativa para estudar determinado fenômeno e seu desenrolar em construção de teoria, a partir do estudo de caso. A pergunta de pesquisa deve ser clara, de grande importância para as organizaçôes e teorias, enfatizando que não há uma teoria existente para uma resposta plausível e iniciar a pergunta utilizando "como" ou "por que" (YIN, 2005; EISENHARDT; GRAEBNER, 2007; LIMA et al., 2012; MARQUES et al., 2015).

A construção da teoria tem pontos fortes como possibilidades de inovação de testes, independência de literatura prévia à observação empírica e validade empírica oriunda das ligações internas com as evidências empíricas da área ou de outras áreas, cuja teoria existente não é adequada, além de complementar a teoria incremental de construção de pesquisas da ciência (EISENHARDT, 1989; EISENHARDT; GRAEBNER, 2007; GIBBERT et al., 2008; EISENHARDT et al., 2016).

A construção teórica de casos únicos ou múltiplos segundo Eisenhardt (1989), Eisenhardt e Graebner (2007), Gibbert et al. (2008) e Eisenhardt et al. (2016) perpassam pelo desenvolvimento de teoria indutiva. A teoria emergente baseia-se no reconhecimento pelos padróes de relaçóes de triangulação intracaso e intercaso. Considera-se o cerne do estudo a replicação lógica dos dados e cada caso tem a sua própria base analítica (GIBBERT; RUIGROK, 2010; YIN, 2014). Os achados mais relevantes são encontrados em pesquisas qualitativas originárias de pesquisa dedutiva. A lógica indutiva e dedutiva é complementar para a construção da teoria de casos, sendo que a teoria indutiva gera nova teoria a partir dos achados e a teoria dedutiva testa esses achados.

Para Larrinaga (2017) e Yin (1981, 2014), por meio da perspectiva tradicional, o estudo de caso exibe uma narrativa excessiva, que não tem uma estrutura previsível, difícil de ler e escrever e nos casos de narrativa é possível substituir a resposta por uma série de perguntas abertas. Para o desenvolvimento das narrativas, os pesquisadores fazem: uma escrita bem apurada dos elementos das entrevistas individuais; reunióes específicas ou grandes eventos; anotações de atividades diárias ou semanais; e resumos de relatórios ou documentos individuais. Cada unidade da narrativa precisa demonstrar a relevância dos dados em diferentes elementos, para serem gravados no lugar de notas de narração. No estudo de caso explicativo é necessária uma representação exata dos fatos do caso, explicações alternativas dos fatos devem ser fomentadas e deve-se ter uma conclusão com base na única explicação que aparece mais equivalente com os fatos. 
A análise do caso constitui um processo complexo que determina a objetividade do pesquisador, porém não fica livre de inconsistências (YIN, 1981; MOZZATO; GRZYBOVSKI; TEIXEIRA, 2016) e pode ser feita por meio de um relato ou um resumo individual, mas objetivando dirimir conflitos e sugerindo que os casos sejam agregados. As limitaçóes do estudo de caso decorrem quando os fatores são isolados dentro de caso particular, sendo que outra limitação é o grande número de casos suficientes para justificar um corte transversal para tabulação. Nos casos de levantamento de caso ou comparação de casos, o pesquisador deve aguardar as provas para passar à etapa da análise (YIN, 1981).

Outro questionamento de alguns pesquisadores é a seleção dos casos que devem ser representativos para ser generalizados e, como resposta, o pesquisador deve deixar claro que o objetivo é desenvolver a teoria e não a testar. Por isso, a coleta de dados deve ser adequada para esclarecer e estender os relacionamentos e a lógica entre os constructos (EISENHARDT; GRAEBNER, 2007; LARRINAGA, 2017). O pesquisador deve aterse em não conflitar dados qualitativos com pesquisa qualitativa. Os dados qualitativos beneficiam o aprofundamento das entrevistas e outros instrumentos e a pesquisa qualitativa usa esses dados para descrever a construção social e expandir a teoria. Ao desenvolver estudo de casos múltiplos, o pesquisador precisa atentar para as limitaçóes geográficas, a fim de enriquecer o trabalho e não se perder diante do volume das informaçóes durante as análises. Outro quesito é não confundir a definição de construção de teoria, que é puramente criar teoria, observando os padrōes dentre os dados sistematizados dos dados empíricos coletados (EISENHARDT; GRAEBNER, 2007).

Para Ridder et al. (2009), a contribuição teórica pode originar-se dos conceitos teóricos para um novo contexto, desde que cuidadosamente definida e descrita pelo pesquisador. Os pesquisadores devem avançar além dos limites das configuraçóes teóricas existentes e a contribuição teórica pode surgir no entrelaçamento dos conceitos teóricos e nas suas relações em diferentes contextos empíricos.

Para a criação de teoria a partir do estudo de caso único, é mais limitado e complicado do que em casos múltiplos. A pesquisa deve ser indutiva e a teoria surge como padróes de relacionamentos entre constructos reconhecidos no caso. No estudo de caso único, o caso deve ser "extraordinariamente revelador" (MARIOTTO et al., 2014, p. 361), exemplar ou quando ofertar oportunidades para pesquisa de acesso incomum. Essas oportunidades do caso podem acrescentar ajustes em um conhecimento já cristalizado da realidade (MARIOTTO et al., 2014, MARQUES et al., 2015; LARRINAGA, 2017).

$\mathrm{Na}$ falsificação ou refinamento da teoria, a falsificação requer um processo com mais rigor e pode estar sujeita a uma proposta teórica, sendo necessária uma proposição falsa para desconstruir um argumento. $\mathrm{O}$ estudo de caso é um método que favorece a falsidade teórica em decorrência do aprofundamento do caso investigado (MARIOTTO et al., 2014).

A criação de teoria segundo Welch et al. (2011), oriunda do estudo de caso, apresenta quatro tipos, que são: construção de teoria indutiva, interpretação sensorial, experiência natural e explicação do contexto. A pesquisa qualitativa suporta a conotação que é sensível ao contexto, levando a descontextualização, a qual configura-se em um desafio: se teorizar é generalizar o contexto, explicar e contextualizar sáo definitivamente opostos. Os autores relatam que em uma situação contingencial, a associação de um mecanismo causal leva a 
produção de um resultado e, assim, explica um fenômeno tal como ele é. Partindo de uma explicação causal, as ponderaçóes sobre o fenômeno começam a ter diferencial mundial. Em seus estudos de 10 anos, utilizando os bancos de dados do Journal of International Business Studies, Academy of Management Journal e Journal of Management Studies, encontraram que a maioria dos artigos tratou de construção de teoria indutiva, o que instiga o uso de outros métodos de teorização para aumentar a robustez e, logo, o poder de explicaçáo e a contextualização potencial do estudo de caso. Thomas (2011) propõe outra classificação, sendo: a) o sujeito do estudo a que corresponde o caso em si; e b) o objeto do estudo a que se refere a análise ou a teoria, com base na qual o assunto será explicado. Portanto, o estudo pode ser focalizado na teoria ou no estudo ilustrativo.

As oportunidades fundamentais do estudo de caso qualitativo perpassam por um bom embasamento teórico, identificando lacunas e proposiçôes para resolvê-las e justificando o porquê da escolha em detrimento dos testes existentes. $\mathrm{O}$ design com o uso de diagramas, ou o resumo com caixas e setas deixa mais claro o que se pretende fazer na escrita da teoria. $\mathrm{O}$ estudo de caso pode envolver vários instrumentos de pesquisa como entrevistas, arquivos de dados, pesquisa de dados, etnografia e observações. As entrevistas são uma fonte inesgotável de captação de informaçôes de acordo com o fenômeno em estudo, porém, os vieses devem ser evitados. O número de entrevistados deve ser substancial e envolver diversos sujeitos, deve-se conhecer bem o tema da pesquisa para propiciar abertura de novas perspectivas e deve-se relacionar o passado e o presente, a fim de que haja maior profundidade sobre o fenômeno (YIN, 2014; EISENHARDT; GRAEBNER, 2007; MARQUES et al., 2015; LARRINAGA, 2017).

Os autores (GIBBERT; RUIGROK, 2010; LIMA et al., 2012 ; MARQUES et al., 2015; LARRINAGA, 2017) sugerem que os critérios de qualidade - validade do constructo, validade interna / externa e confiabilidade - devem ser adotados pelos pesquisadores. Devem ser citados os documentos utilizados e as açóes tomadas para garantir o rigor do método. Devem ser relacionadas detalhadamente as estratégias para garantir a validade do constructo e as validades interna e externa. As estratégias contingenciais devem ser citadas e como os reveses e imprevistos foram sanados para manter os procedimentos planejados anteriormente. Essas práticas objetivam maior transparência ao leitor, facilitando a tomada de decisão.

\section{CONSIDERAÇÓES FINAIS}

Considerando-se que o estudo de caso é estigmatizado por alguns pesquisadores internacionais no âmbito da gestão e muito utilizado pelos brasileiros, presume-se que essa estratégia de pesquisa necessita de bom delineamento e parâmetros de qualidade, desde o levantamento do problema até o encerramento da pesquisa.

O preconceito sobre o estudo de caso deve ser desconstruído antes de se fazer a opçáo por essa estratégia, pois os argumentos principais referem-se à falta de generalização dos resultados e a discussão dos dados. Isso leva a reflexão de que, ao escolher a estratégia de estudo de caso, o pesquisador deve aprofundar seus conhecimentos sobre o-esse tipo de estudo, conhecer seus desafios e garantir a confiabilidade e validade externa. O preconceito sobre o uso desse método tem minimizado nos últimos anos, porém, percebe-se ainda 
discriminação em meios acadêmicos internacionais, haja vista visão dominante positivista. E tratando-se de estudo de caso único, o desafio é mais genuíno e quando se objetiva gerar teoria ou aprimorar uma teoria pré-existente.

Este artigo proporcionou um diálogo entre as diversas vertentes de pesquisadores, com a proposta de buscar o devido mérito ao método estudo de caso qualitativo.

O objetivo deste ensaio foi minimizar o motivo que leva ao estigma do estudo de caso no meio acadêmico. Percebe-se que existe um dualismo e falta de posicionamento entre os cientistas sociais, mas os autores apontam diretrizes para um bom estudo de caso, que perpassa pelo problema e pergunta a serem investigados, métodos, passos para a coleta de dados, triangulaçáo dos achados, uso dos critérios de qualidade e conclusão.

Um dos dilemas é a questão da generalização dos achados que leva à dicotomia entre os autores, pois alguns pesquisadores limitam o produto final do estudo de caso, que não pode ser generalizado devido às diferenças do contexto do estudo, seja histórico ou socioeconômico cultural. Outros pesquisadores argumentam que os estudos de casos podem ser generalizados nos casos gerais. A generalização dos achados de estudos de casos, na pesquisa quantitativa, necessita usar elementos genéricos em grandes investigaçóes.

O estigma dos pesquisadores decorre da comparação do estudo de caso com outras estratégias, principalmente em relação à generalização e à construção de teoria. Foram elencados os principais desafios constantes da literatura usada no estudo e as oportunidades segundo Yin (2005, 2014), Eisenhardt e Graebner (2007), Ridder et al. (2009), Gibbert e Ruigrok (2010), Welch et al. (2011), Lima et al., (2012), Mariotto et al. (2014), Marques et al., (2012), Harrison et al. (2017) e Larrinaga (2017).

Portanto, para legitimar o estudo de caso qualitativo é necessária implementar uma abordagem de qualidade, que atenda aos quatro ou três critérios de rigor acadêmico, dando representatividade ao caso. No entanto, o pesquisador não positivista pode optar por não atender os critérios explicitados no Quadro 3 e deixar que o leitor possa generalizar as conclusóes do seu achado em casos semelhantes de acordo com o contexto e, assim, gerar um conhecimento pessoal. Diante das inúmeras técnicas de evidência para a construção do estudo de caso, o pesquisador deve atentar para selecionar as fontes mais apropriadas para o seu caso, evitando perder tempo e esforço.

As limitações do estudo decorrem do número reduzido de periódicos internacionais da administração, que aplicam esta metodologia e também ao preconceito ao seu uso pelos pesquisadores brasileiros.

As contribuiçóes deparam no passo a passo para o desenvolvimento de um bom paper qualitativo, aplicando os critérios de qualidade citados acima e a possibilidade de desenvolver a teoria ou a sua construção. A triangulação do produto final do estudo de caso pode ser realizada com outros produtos similares e contraditórios e apresenta a possibilidade de generalização dos achados na gestão. 


\section{AGRADECIMENTO}

Ao Professor Doutor José Márcio de Castro da PUCMINAS pelo apoio e incentivo no desenvolvimento deste estudo.

\section{REFERÊNCIAS}

BONOMA, T.V. Case Research in Marketing: opportunities, problems, and a process. Journal of Marketing Research, v. XXII, 1985.

CASTRO, J.M.; REZENDE, S. F.L. Validade e confiabilidade de estudos de casos qualitativos em gestão publicados em periódicos nacionais. Organizaçóes em Contexto, v.4, n.28, p. 29-42, jun./dez.2018.

CEPEDA, G.; MARTIN, D. A review of case studies publishing in Management Decision 2003-2004: guides and criteria for achieving quality in qualitative research. Management Decision, v.43, n.6, p. 851-876, 2005.

DE MASSIS, A.; KOTLAR, J. The case study method in family business research: Guidelines for qualitative scholarship. Journal of Family Business Strategy, v.5, n. 1, p. 15-29, 2014.

EISENHARDT, K.M. Building Theories from Case Study Research. Academy of Management Review, v.14, n.14, p. 532-550, 1989.

EISENHARDT, K.M.; GRAEBNER, M.E. Theory building from cases: opportunities and challenges. Academy of Management Journal, v. 50, n. 1, p.25-32, 2007.

EISENHARDT, K. M.; GRAEBNER, M.E.; Sonenshein, Scott. From the editors: Grand Challenges and Inductive Methods: Rigor without Rigor Mortis. Academy of Management Journal, v. 59, n.4, p. 1113-1123,2016. Doi: 10.5465/amj.2016.4004

FLYVBJERG, B. Five misunderstandings about case-study research, Qualitative Inquiry, v.12, n.2, p. 219-245,2006.

FREITAS, W.R.S.; JABBOUR, C.J.C. Utilizando estudo de caso(s) como estratégia de pesquisa qualitativa: boas práticas e sugestôes. Estudo \& Debate, Lajeado, v.18, n.2, p.722, 2011.

GIBBERT, M.; RUIGROK, W.; WICKI, B. What passes as a rigorous case study? Strategic Management Journal, v.29, n.3, p.1465-1474,2008.

GIBBERT, M.; RUIGROK, W. The "what" and "how" pf case study rigor: three strategies based on published work. Organization Research Methods, v.13, n.4, p.710737,2010 . 
JICK, T.D. Mixing Qualitative and quantitative Methods: triangulation in action. Administrative Science Quartely, v. 24, n.4, p. 602-11, 1979.

HARRISON, H.; BIRKS, M.; FRANKLIN, R.; MILLS, J. Case study research: foundations and methodological orientations. Forum Qualitative Sozialforschung / Forum: Qualitative Social Research, [S.1.], v. 18, n. 1, jan. 2017.

LARRINAGA, O.V. Is it desirable, necessary and possible to perform research using case studies? Cuadernos de Gestión, v.17, n.1, p.147-172, 2017. DOI: 10.5295/ cdg. 140516 ov

LEE, A.S. A Scientific methodology for MIS case studies. MIS Quartely, v.13, p.33-50, 1989.

LEONARD-BARTON, D. A Dual Methodology for Case Studies: Synergistic Use of a Longitudinal Single Site with Replicated Multiple Sites. Organization Science, 1, 3, Special Issue: Longitudinal Field Research Methods for Studying Processes of Organizational Change, p. 248-266, 1990.

LIMA, J.P.C.; ANTUNES, M.T.P.; MENDONÇA NETO, O.R.; PELEIAS, I.R. Estudos de caso e sua aplicação: proposta de um esquema teórico para pesquisas no campo da contabilidade. Revista de Contabilidade e Organizaçóes, v.6, n.14, p.127-144, 2012.

LLEWELLYN, S.; NORTHCOTT, D. The "singular view" in management case studies qualitative research in organizations and management. An International Journal, v. 2, n. 3, p.194-207, 2007.

MARIOTTO, F. L., Zanni, P. P.; Moraes, G.H.S.M. What is the of a single-case study in management research. Revista de Administraçáo de Empresas; v.54, n.4, p. 358-369, jul./ago. 2014. Doi.org/10.1590/S0034-759020140402

MARQUES, K.C.M.; CAMACHO, R.R.; ALCÂNTARA, C.C.V. Assessment of the methodological rigor of case studies in the field of management accounting published in journals in Brazil. Revista Contabilidade \& Finanças-USP, v. 26, n. 67, p.27-42, janeiro a abril 2015. Doi: 10.1590/1808-057x201500280

MARTINS, G.A. Estudo de caso: uma reflexão sobre a aplicabilidade em pesquisas no Brasil. Revista de Contabilidade e Organizaçóes, v. 2, n. 2, p. 9-18, jan./abr. 2008.

MOHD NOOR, K. B. Case study: A strategic research methodology. American journal of applied sciences, v.5, n.11,p. 1602-1604,2008.

MOZZATO, A.R.; GRZYBOVSKI, D.; TEIXEIRA, A.N. Análises qualitativas nos estudos organizacionais: as vantagens no uso do software $\mathrm{NVIVO}^{\circ}$. Revista Alcance Eletrônica, v.23, n.4, p.578-587, out./dez. 2016. Doi: alcance.v23n4.p578-587 
PLATT, J. Case study. In: Outhwaite, W. \& Turner, S. The sage handbook of social science methodology. London: Sage, 2007.

RAGIN, C.C.; BECKER, H.S. What's a case? Exploring the foundations of Social Inquiry. UK, Cambridge: Cambridge University Press, p. 1-17, 1992.

RIDDER, H.G; HOON, C.; BALUCH, A.MC. The theoretical contribution of case study research to the field of strategy and management. Research methodology in strategy and management, v.5, p.137-175, 2009. / Doi: 10,1108 / S1479-8387 (2009) 0000005007.

RUZZENE, A. Drawing lessons from case studies by enhancing comparability.

Philosophy of the Social Sciences, v.42, n.1, p. 99-120,2012.

SAYER, A. Realism and social Science. London: Sage, 2000.

STAKE, R.E. The art of case study research. Thousand Oaks: Sage, 1995.

STAKE, R.E. Case Studies, in: Norman K. Denzin and Yvonna S. Lincoln (Eds),Handbook of Qualitative Research, 2. ed, Thousand Oaks, CA: Sage, p. 43554,2000 .

THOMAS, G. A typology for the case study in social science following a review of definition, discourse, and structure. Qualitative Inquiry, v.17, n.6, p.511-521,2011.

TSANG, E. W. K. Generalizing from research findings: The merits of case studies. International Journal of Management Reviews, v.16, n.4, p. 369-383,2014.

VOSS, C.; TSIKRIKTSIS, N.; FROHLICH, M. Case research in operations management. International Journal Of Operations \& Production Management, v. 22, n. 2, p. 195-219, 2002.

YIN, R.K. The Case Study Crisis: some answers. Administrative Science Quartely, Cornell University, v.26, p.58-65, 1981.

YIN, R.K. Case study research: design and methods. Beverly Hills, CA: Sage publications, 1984.

YIN, R.K. Pesquisa estudo de caso: Projeto e métodos. 3. ed. Thousand Oaks, CA: Sálvia, 2003.

YIN, R.K. Estudo de caso: planejamento e métodos. 2. ed. Porto Alegre: Bookman, 2005.

YIN, R.K. Case studies: design and methods, 4.ed,Thousand Oaks: Sage publications, 2009. 
YIN, R.K. Validity and generalization in future case study evaluations. Evaluation, v.19, n.3, p. 321-332,2013. Doi: 10.1177/1356389013497081.

YIN, R. K. Case study research: Design and methods. Los Angeles, CA: Sage, 2014.

WELCH, C.; PIEKKARI, R.; PLAKOYIANNAKI, E.; PAAVILAINEN-MÄNTYMÄKI, E. Theorising from case studies: Towards a pluralist future for international business research. Journal of International Business Studies, v. 42, p.740-762, 2011.

ZAPPELLINI, M.B.; FEUERSCHÜTTE, S.G. O uso da triangulação na pesquisa científica brasileira em administração. Administração: ensino e pesquisa, Rio de Janeiro, v.16, n.2, p.241-273, Abr / Mai / Jun 2015. Doi: 10.13058/raep.2015.v16n2.238. 